\title{
Patient, Caregiver, and Clinician Perspectives on Expectations for Home Healthcare after Discharge: A Qualitative Case Study
}

\author{
Christine D Jones, MD, MS'; Jacqueline Jones, PhD, RN, FAAN²; Kathryn H Bowles, PhD, RN, FAAN, FACMI3,4;

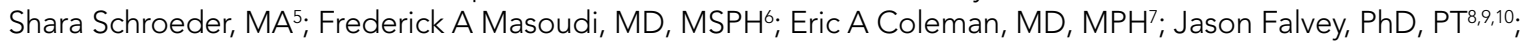 \\ Cari R Levy, MD, PhD7,9; Rebecca S Boxer, MD, MS 9.11
}

\begin{abstract}
'Division of Hospital Medicine, Department of Medicine, University of Colorado Anschutz Medical Campus, Aurora, Colorado; ${ }^{2}$ College of Nursing, University of Colorado Anschutz Medical Campus, Aurora, Colorado; ${ }^{3}$ School of Nursing, University of Pennsylvania, Philadelphia, Pennsylvania; ${ }^{4}$ Center for Home Care Policy and Research, Visiting Nurse Service of New York, New York, New York; ${ }^{5}$ College of Liberal Arts and Sciences, Department of Sociology, University of Colorado, Denver, Colorado; ' ${ }^{D}$ ivision of Cardiology, University of Colorado Anschutz Medical Campus, Aurora, Colorado; ' Division of Health Care Policy and Research, University of Colorado Anschutz Medical Campus, Aurora, Colorado; ${ }^{8}$ Physical Therapy Program, Department of Physical Medicine and Rehabilitation, University of Colorado Anschutz Medical Campus, Aurora, Colorado; ${ }^{9}$ Veterans Affairs Medical Center, Denver, Colorado; ${ }^{10}$ Yale School of Medicine, New Haven, Connecticut; "Division of Geriatric Medicine, University of Colorado Anschutz Medical Campus, Aurora, Colorado.
\end{abstract}

BACKGROUND: Patients discharged from the hospital with skilled home healthcare $(\mathrm{HHC})$ services have multiple comorbidities, high readmission rates, and multiple care needs. In prior work, HHC nurses described that patients often express expectations for services beyond the scope of skilled HHC.

OBJECTIVE: The objective of this study is to evaluate and compare expectations for $\mathrm{HHC}$ from the patient, caregiver, and $\mathrm{HHC}$ perspectives after hospital discharge.

DESIGN/PARTICIPANTS: This was a descriptive qualitative case study including $\mathrm{HHC}$ patients, caregivers, and clinicians. Patients were discharged from an academic medical center between July 2017 and February 2018.

RESULTS: The sample ( $\mathrm{N}=27$ ) included $11 \mathrm{HHC}$ patients, eight caregivers, and eight HHC clinicians (five nurses and three physical therapists). Patient mean age was 66 years and the majority were female, white, and had Medicare. We observed main themes of clear and unclear expectations for $\mathrm{HHC}$ after discharge. Clear expectations occur when the patient and/or caregiver have expectations for $\mathrm{HHC}$ aligned with the services received. Unclear expectations occur when the patient and/or caregiver expectations are uncertain or misaligned with the services received. Patients and caregivers with clear expectations for $\mathrm{HHC}$ frequently described prior experiences with skilled HHC or work experience within the healthcare field. In most cases with unclear expectations, the patient and caregiver did not have prior experience with $\mathrm{HHC}$.

CONCLUSIONS: To improve HHC transitions, we recommend actively engaging both patients and caregivers in the hospital and $\mathrm{HHC}$ settings to provide education about $\mathrm{HHC}$ services, and assess and address additional care needs. Journal of Hospital Medicine 2019;14:90-95. () 2019 Society of Hospital Medicine atients who are discharged from the hospital with home healthcare $(\mathrm{HHC})$ are older, sicker, and more likely to be readmitted to the hospital than patients discharged home without $\mathrm{HHC} .{ }^{1-3}$ Communication between clinicians in different settings is a key factor in successful transitions. In prior work, we focused on communication between primary care providers, hospitalists, and $\mathrm{HHC}$ nurses to inform efforts to improve care transitions. ${ }^{4,5}$ In one study, $\mathrm{HHC}$ nurses described that patients frequently have expectations beyond the scope of what skilled HHC provides, ${ }^{5}$ which prompted us to also question experiences of patients and caregivers after

*Corresponding Author: Christine D. Jones, MD, MS; E-mail: christine.jones@ ucdenver.edu; Telephone: 720-848-4289; Twitter: @jones_delong

Received: August 3, 2018; Revised: November 16, 2018 ;

Accepted: December 6, 2018

๑ 2019 Society of Hospital Medicine DOI 10.12788/jhm.3140 discharge with skilled HHC (eg, nursing and physical therapy).

In a prior qualitative study by Foust and colleagues, $\mathrm{HHC}$ patients and caregivers described disparate experiences around preparation for hospital discharge-patients expressed knowing about the timing and plans for discharge, and the caregivers frequently felt left out of this discussion. ${ }^{6}$ In other studies, caregivers of recently discharged patients have described feeling excluded from interactions with clinicians both before and after discharge..$^{78}$ In another recent qualitative study, caregivers described uncertainty about their role compared with the $\mathrm{HHC}$ role in caring for the patient. ${ }^{9}$

As of 2016, a majority of states had passed the Caregiver Advise, Record, and Enable (CARE) Act, which requires hospitals to (1) record a family caregiver in the medical record, (2) inform this caregiver about discharge, and (3) deliver instructions with education about medical tasks that they will need to complete after discharge..$^{10}$ In the context of the CARE Act, hospitals are encouraged to increase caregiver engagement to prepare for 
TABLE 1. Demographics for Patients, Caregivers, and Home Healthcare (HHC) Clinicians

\begin{tabular}{|c|c|c|c|}
\hline & Patient $(n=11)$ & Caregiver $(n=8)$ & HHC Clinician $(n=8)$ \\
\hline \multicolumn{4}{|l|}{ Race/Ethnicity } \\
\hline White & $73 \%$ & - & - \\
\hline \multicolumn{4}{|l|}{ Insurance } \\
\hline Medicare & $73 \%$ & - & - \\
\hline Medicaid & $9 \%$ & - & - \\
\hline Private & $9 \%$ & - & - \\
\hline
\end{tabular}

discharge, but it is unclear whether this engagement is occurring for patients in general and $\mathrm{HHC}$ patients in particular. Because more than $80 \%$ of $\mathrm{HHC}$ patients have a primary caregiver outside of $\mathrm{HHC}$, caregiver engagement around the time of discharge could be a key factor in care transitions. ${ }^{11}$

The objective of this study is to evaluate and compare expectations for $\mathrm{HHC}$ from the patient, caregiver, and $\mathrm{HHC}$ perspectives after hospital discharge. By combining all three groups into a case study, we aim to build on our prior work with $\mathrm{HHC}$ nurses to explore how expectations for $\mathrm{HHC}$ compare within and across cases of patients, caregivers, and $\mathrm{HHC}$ clinicians.

\section{METHODS}

\section{Study Design}

In this qualitative descriptive case study, we interviewed $\mathrm{HHC}$ patients, an involved caregiver, and the $\mathrm{HHC}$ clinician completing the first $\mathrm{HHC}$ visit within 7-14 days following hospital discharge. We chose this timeframe to allow patients to receive one or more $\mathrm{HHC}$ visits following hospital discharge.

\section{Population}

A convenience sampling strategy was employed to recruit a sample that would reflect a national sample of Medicare $\mathrm{HHC}$ patients based on age, sex, race, and ethnicity. Because a majority of $\mathrm{HHC}$ users in the United States are Medicare beneficiaries $\geq 65$ years old, ${ }^{12}$ eligibility was initially limited to patients $\geq 65$ years old. Due to recruitment challenges, the age range was broadened to $\geq 50$ years old in October 2017 . Because our goal was to better understand the experience of general medicine patients with multiple comorbidities, we recruited patients from one general medicine unit at an academic hospital in Colorado. Patients on this unit were screened for eligibility Monday-Friday (excluding weekends and holidays) based on research assistant availability.

Criteria included are as follows: HHC referral, three or more comorbidities, resides in the community prior to admission (ie, not in a facility), cognitively intact, English speaking, and able to identify a caregiver participating in their care. Eligible patients were approached for written consent prior to discharge to allow us to contact them 7-14 days after discharge for an interview by phone or in their home, per their preference. At the time of consent, patients provided contact information for their informal caregiver. Caregiver eligibility criteria included the following: age $\geq 18$ years and provides caregiving at least one hour a week before hospital discharge. HHC clinicians approached for interviews had completed the first $\mathrm{HHC}$ visit for the patient following discharge. Both caregivers and $\mathrm{HHC}$ clinicians provided verbal consent for interviews. All participants received a $\$ 25$ gift card for participation in the study.

\section{Framework and Data Collection}

Our interview guides were organized by the Agency for Healthcare Research and Quality Care Coordination Framework, an approach we have taken in prior work. $4,5,13$ We added questions about patient preparation and self-management support to build on findings from a prior study with $\mathrm{HHC}$ nurses and on prior work by Coleman and colleagues. ${ }^{5,14}$ Sample questions from the interview guides for patients, caregivers, and $\mathrm{HHC}$ clinicians within key analysis domains are included in Appendix 1. The patient and caregiver interviews were completed by an individual with prior experience in social work and healthcare (SS). The HHC clinician interviews were completed by either this individual (SS) or a physician-researcher with experience in qualitative methods (CJ). Patients and caregivers could choose to be interviewed individually or together. All interviews were digitally recorded and transcribed verbatim.

\section{Analysis}

This study aimed to evaluate the clarity of expectations related to HHC after discharge within and across cases. We primarily explored domains of patient preparation, assessing needs and goals, and creating a plan of care for skilled HHC from patient and caregiver perspectives. Because qualitative work had been completed previously with HHC clinicians, HHC perspectives were used primarily for triangulation of perspectives 
TABLE 2. Clear and Unclear Expectations within and across Cases: Quotes from Patients, Caregivers, and Home Healthcare Clinicians

\begin{tabular}{|c|c|c|c|}
\hline Case \# & Patient & Caregiver & HHC Clinician \\
\hline \multicolumn{4}{|c|}{ Clear Expectations across Cases } \\
\hline 1 & $\begin{array}{l}\text { Patient: In home care, you're in your own home... You're comfortable. } \\
\text { They'll recommend how you should have your home so you don't feel } \\
\text { afraid and I think that's important. }\end{array}$ & $N A$ & $\begin{array}{l}\text { HHC RN: Oh yes... she's very prepared for it (HHC). } \\
\text { She has had a wound ... for over ten years we've been } \\
\text { trying to get healed... So she knows home healthcare } \\
\text { well. She prefers home healthcare just because she's } \\
\text { more comfortable in her own home. }\end{array}$ \\
\hline 2 & $\begin{array}{l}\text { Patient: I think it (the transition to } \mathrm{HHC} \text { ) was made fairly easy for me. } \\
\text { Of course myself being in the health field, my daughter being in the } \\
\text { health field ... helps a lot too because, you know, we both know } \\
\text { what to expect. We both, you know, know what we should be doing. }\end{array}$ & $\begin{array}{l}\text { Caregiver: ....yeah, my role is pretty well defined and as } \\
\text { a family we've all got our roles pretty well defined as well } \\
\text { so that helps... they do like physical therapy and those } \\
\text { types of things and we take care of everything else so it's } \\
\text { been a great relationship. }\end{array}$ & $\begin{array}{l}\text { HHC PT: ...I think (the patient is) very prepared of what's } \\
\text { coming... she knows what's ahead of her. }\end{array}$ \\
\hline 3 & $\begin{array}{l}\text { Interviewer: ... have you been surprised at all by what home health } \\
\text { is able to help you with or not able to help you with? } \\
\text { Patient: Not really. I'm so... it's cool. I like it. }\end{array}$ & $\begin{array}{l}\text { Caregiver: I don't know...I can't look at his leg and say } \\
\text { this is the appropriate color of red or it's not...or it's too } \\
\text { swollen... Only a nurse can. So if I know (the HHC nurse) } \\
\text { is coming to check on it, it does make me feel better } \\
\text { knowing that I don't have to worry quite so much about } \\
\text { it being, you know, beyond my control. }\end{array}$ & $\begin{array}{l}\text { HHC RN: ...I think he's adjusting well. He has the } \\
\text { support of his (caregiver) for sure but, you know ... } \\
\text { she works a full time job so I think ... his expectations } \\
\text { are realistic. }\end{array}$ \\
\hline
\end{tabular}

Unclear Expectations across Cases

$4 \quad$ Patient:Well, um...I don't know...I didn't expect it to happen but um...

Interviewer: You didn't expect home health to happen? Patient: Yeah.
Caregiver: Well, one of the things that I was wondering about is that whether she would help her with her shower.

...I think one of the things is that we don't know exactly what the home healthcare is supposed to do.
HHC RN: ...I don't feel like (the patient) was totally clear on why we were there in the first place so... I don't think she really had any expectations.
Patient: And so they (the HHC agency) find out I'm in the hospital so as soon as I come home, I get all kind of telephone calls. They say ...I'm physical therapist. I'm going to come and see you and I say, 'For what? We just talked to you at hospital' so I didn't know what to do.

\section{NA}

HHC PT: ....you know, she kind of didn't seem like she knew like, you know, why I was there and this and... it took her a long time when I was in the house to just sit down and get started..
Patient: ... I had never had home healthcare before. It was very reassuring. I was a little uncertain, like it was designed for someone in more of a chronic debilitated state than me but I think I just didn't know much about it, you know, so I thought wow, I don't need these people coming forever, but indeed they're not coming forever.
HHC RN: You know, she didn't quite know what I was going to do. She knew I was coming. Actually she did. I take it back, she did kind of know that I was going to be there to show her what to do, how's that?

Abbreviations: $\mathrm{HHC}$, home healthcare; RN, registered nurse; NA, not applicable; PT, physical therapist.

about expectations where possible. The analysis team was composed of the two interviewers (SS and CDJ) and a qualitative methods expert $(\mathrm{JJ})$. We used our established team-based inductive approach to develop themes around patient expectations and preparation for $\mathrm{HHC}$, with deductive connections to the framework as applicable. ${ }^{15,16}$ Two team members completed the initial coding after every one to three interviews to ensure the themes were developing iteratively. Group discussions including the third team member were used to resolve discrepancies and to complete a team-based iterative analysis until informational saturation for expectations after discharge was reached from the patient and caregiver perspectives (ie, no new codes were identified). ${ }^{17}$ Once the team reached informational saturation with codes, we recruited three additional patients to ensure no new codes were identified in key domains before concluding recruitment. ATLAS.ti version 7.5.17 (ATLAS.ti Scientific Software Development GmbH, Berlin, Germany) was used to facilitate coding and analysis. This study was approved by the Colorado Multiple Institutional Review Board (protocol 17-0553).

\section{RESULTS}

Between July 2017 and February 2018, patients were recruited for participation in this study. Because the discharge destination plans could change multiple times in a hospitalization, the eligibility of patients for the study could change throughout hospitalization. To give further context about patients on this unit during the study timeframe, we completed a retrospective review of the 1,024 patient discharges from the unit and found that 38 patients met the eligibility criteria. Overall, 15 patients provided written consent (11 women and four men), and 11 completed interviews. The remaining four were unable to complete interviews due to a change in postdischarge plans that no longer included $\mathrm{HHC}$ (two patients) and hospital readmissions prior to interviews (two patients). In total, interviews were completed with 27 individuals: 11 patients, eight caregivers, and eight $\mathrm{HHC}$ clinicians (five nurses and three physical therapists). For five of the interviews, the patient and the caregiver requested to be interviewed together. In four cases, interviews were missing from the caregiver (one case), the $\mathrm{HHC}$ clinician (one case), or both the caregiver and the HHC clini- 
cian (two cases). Overall, perspectives were available from the complete triad of patients, caregivers, and HHC clinicians in seven cases, and perspectives were available from the patient and at least one other individual (ie, caregiver or HHC clinician) in two additional cases.

Patient interviews lasted an average of 43 minutes, caregiver interviews an average of 41 minutes, and $\mathrm{HHC}$ clinician interviews an average of 25 minutes. Patients were on average 66 years old (range 52-85 years), and most were women and white. Six of the patients had prior experience with $\mathrm{HHC}$ services, and five were new HHC patients. Primary diagnoses for patients included the following: sepsis (three cases), urinary tract or kidney infections (two cases), bone/hardware infections (two cases), Clostridium difficile infection (one case), acute respiratory failure with hypoxia (one case), aortic stenosis (one case), and acute pancreatitis (one case). For caregivers, the average age was 61 years, all were women, and they had a spouse or other family member in six cases and a nonfamily caregiver in two cases. HHC clinicians were an average of 40 years old, half were women, and the average time providing HHC was 4.4 years (Table 1 ).

We observed the two main themes of clear and unclear expectations for $\mathrm{HHC}$ after discharge. Clear expectations occur when the patient and/or caregiver have expectations for $\mathrm{HHC}$ that align with the services they receive. Unclear expectations occur when the patient and/or caregiver expectations are either uncertain or misaligned with the services they receive. Although not all interviews yielded codes about clear or unclear expectations, patients described clear expectations in five cases and unclear expectations in another five cases.

In nine cases with more than one perspective available, expectations were compared within cases and found to be clear (three cases), unclear (three cases), or discordant (three cases) across perspectives. For the discordant cases, the description of clear and unclear expectations differed between patients and either their caregiver or their $\mathrm{HHC}$ clinician. Patients and caregivers with clear expectations for $\mathrm{HHC}$ frequently described prior experiences with skilled HHC or work experience within the healthcare field. In most cases with unclear expectations, the patient and caregiver did not have prior experience with $\mathrm{HHC}$. In addition, the desire for assistance with personal care for patients such as showering and housekeeping was described by caregivers with unclear expectations. The results are organized into clear, unclear, and discordant expectations from the perspectives of patients, caregivers, and $\mathrm{HHC}$ clinicians within cases.

\section{Clear Expectations within Cases}

Clear expectations for HHC were identified across perspectives in three cases, with sample quotes provided in Table 2. In the case of patient 1, the patient and HHC nurse had known each other for over two years because the patient had a wound requiring long-term $\mathrm{HHC}$ services. A caregiver did not complete an interview in this case. With patient 2, the patient, caregiver, and $\mathrm{HHC}$ physical therapist (PT) all describe that the patient had clear expectations for $\mathrm{HHC}$. In this case, the patient and caregiver describe feeling prepared because of previously receiving $\mathrm{HHC}$, prior work experience in the healthcare field, and a caregiver with experience working in $\mathrm{HHC}$. In the case of patient 3 , the patient had previously received $\mathrm{HHC}$ from the same $\mathrm{HHC}$ nurse.

\section{Unclear Expectations across Cases}

For the three cases in which unclear expectations were described across perspectives, two of the patients described being new to $\mathrm{HHC}$, with representative quotes in Table 2. Patient 4 and her caregiver are new to $\mathrm{HHC}$ and describe unclear expectations for both the $\mathrm{HHC}$ referral and the $\mathrm{HHC}$ role, which was also noted by the $\mathrm{HHC}$ clinician. Of note, the caregiver for patient 4 further described that she was unable to be present for the first $\mathrm{HHC}$ visit. In the case of patient 5, although the patient had previously received $\mathrm{HHC}$, the patient describes not knowing why the HHC PT needs to see her after discharge, which is also noted by the HHC PT. Finally, both patient 6 and her HHC PT describe that the patient was not sure about their expectations for $\mathrm{HHC}$ and that $\mathrm{HHC}$ was a new experience for them.

\section{Discordant Expectation Clarity across Cases}

In three of the cases, the description of clear and unclear expectations was discrepant across roles. In case 7, the caregiver and patient are new to $\mathrm{HHC}$ and express different perspectives about expectations for $\mathrm{HHC}$. The $\mathrm{HHC}$ clinician, in this case, did not complete an interview. The caregiver describes not being present for the first $\mathrm{HHC}$ visit and no awareness that the patient was being discharged with $\mathrm{HHC}$ :

Caregiver: Well, we didn't even know she had home health until she got home.

The same caregiver also expresses unclear expectations for $\mathrm{HHC}$ :

Caregiver: It's pretty cloudy. They (the HHC clinicians) don't help her with her laundry, they don't help with the housekeeping, they don't help... with her showers so somebody is there when she showers. They don't do anything. The only two things like I said is the...home healthcare comes in on Wednesdays to see what she needs and then the therapy comes in one day a week. However, the patient expresses more clear expectations that are being met by $\mathrm{HHC}$.

Patient: They $(\mathrm{HHC})$ have met my expectations. They come in twice a week. They do vitals, take vitals and discuss with me, you know, what my feelings are, how I'm doing and I know they have met my expectations. In case 8, although the patient describes knowing about the HHC PT involvement in her care, she expresses some unclear expectations about an $\mathrm{HHC}$ nurse after discharge.

Patient: As far as home health, I didn't have a real ... plan there at the hospital... They knew about (the HHC PT) coming once a week but as far as, you know, a nurse coming by to check on me, no.

However, the HHC PT describes feeling that the patient had clear expectations for $\mathrm{HHC}$ after discharge: 
Interviewer: Can you reflect on whether she was prepared to receive home healthcare?

HHC PT: Yeah, she was ready.

Interviewer: ... do you feel like she was prepared to know what to expect from you?

HHC PT: Yeah, but I think that comes from being a previous patient also.

Finally, in case 9, the patient describes clear expectations for $\mathrm{HHC}$ even though they were new to $\mathrm{HHC}$ :

Patient: ...I knew what the PT was going to do and ...I still need her because I've lost so much weight so she's been really good, instrumental, at giving me exercises... Occupational therapist...she's going to teach me how to shave, she's going to teach me how to get ready for the day.

The HHC PT describes that although the patient knew the PT role, they reflect that the patient may have been somewhat unclear about expectations for the first $\mathrm{HHC}$ visit:

HHC PT: He knew all that it entailed with the exception of he didn't really know what the first day was going to be like and the first day I don't usually do treatment because it does take a long time to get all the paperwork signed, to do the evaluation and the fact that it takes two hours to do that note.

\section{DISCUSSION}

In this qualitative case study with $\mathrm{HHC}$ patients, caregivers, and clinicians, the participants described varying levels of expectation clarity for $\mathrm{HHC}$ after discharge. We triangulated across and within cases and found three cases with clear expectations and three cases with unclear expectations for $\mathrm{HHC}$ across perspectives. In three additional cases, we found discordant expectations across perspectives: patients and $\mathrm{HHC}$ clinician expectations differed in two of the cases and a patient and caregiver differed in one case. Of interest, in all three cases of clear expectations across perspectives, the patients and/ or caregivers had prior HHC or healthcare work experience. In contrast, in the cases of two caregivers with unclear expectations, neither had prior HHC experience and both described expectations for assistance with personal care or housekeeping. Our findings suggest the need to improve caregiver engagement in HHC decision-making and care delivery, even in the time following the passage of the CARE Act. In addition, our findings suggest that patients and caregivers with unclear expectations for $\mathrm{HHC}$ may benefit from enhanced education about HHC services.

Prior studies in this area have included a qualitative study $\mathrm{HHC}$ patients, caregivers, and clinicians by Foust and colleagues in which multiple caregivers described finding out about the discharge from the patient or other caregivers, rather than being actively engaged by clinicians. ${ }^{6}$ In another recent qualitative study by Arbaje and colleagues, a majority of caregivers described "mismatched expectations" about HHC services, in which caregivers were unclear about their role compared with the $\mathrm{HHC}$ role in caring for the patient. ${ }^{9}$ Of interest, $\mathrm{HHC}$ clinicians in the Arbaje study described one of their key tasks to be "expectation management" for receipt of HHC services. ${ }^{9}$ In our study, the caregivers who described unclear expectations were not able to be present for the first $\mathrm{HHC}$ visit, which may have been a missed opportunity for the HHC clinician to clarify and manage expectations. Overall, findings from each of these studies support that consistent engagement and education from the hospital and $\mathrm{HHC}$ clinicians are needed to prepare patients and caregivers to know what to expect from $\mathrm{HHC}$.

When caregivers have unclear expectations for $\mathrm{HHC}$, they could be expressing the need for more support after hospital discharge, which suggests an active role for hospital teams to assess and address additional support needs with the patients and caregivers. For example, if the patient or caregiver request additional personal care services, a home health aide could help to reduce caregiver burden and improve the support network for the patient. In a prior study in which patients were asked what would help them to make informed decisions about postacute care options, the patients described wanting to receive practical information that could describe how it would apply to their specific situation and perceived needs. ${ }^{18}$ To provide this for patients and caregivers, it would follow that hospitals could provide information about skilled HHC nursing and therapies and information about services that could meet additional needs, such as home health aides.

In the context of the CARE Act, in which hospitals are encouraged to increase family caregiver engagement to prepare for discharge, findings from this and other studies suggest an opportunity to improve caregiver partnership in $\mathrm{HHC}$ transitions. As a result of this work, we recommend intentionally engaging and including caregivers in addition to patients in both the hospital and HHC settings to clarify expectations. Steps to clarify expectations with both patients and caregivers should include the following: (1) providing education and clear expectations for $\mathrm{HHC}$ through verbal interactions and written materials, and (2) assessing and addressing additional needs (eg, personal care) that patients and caregivers may have. To support these efforts, multidisciplinary teams could use previously studied interventions and tools for guidance as they engage caregivers throughout care transitions processes. ${ }^{10,19}$

Limitations of this study include that it was a small qualitative case study of patients, caregivers, and $\mathrm{HHC}$ clinicians from one medical unit at one academic medical center. Most patients in this study had Medicare insurance, were 65 years and older, white, and female. A recent analysis of Medicare $\mathrm{HHC}$ users found that $63 \%$ were female and $75 \%$ were white, which shows that females were overrepresented in our study. ${ }^{1,211}$ The perspective of Black and non-English speaking patients are missing from our study. Finally, we only interviewed individuals in three roles of complex transitions to $\mathrm{HHC}$, and there are likely many additional perspectives on each of these transitions, which could provide additional insights. Results are not generalizable or transferable beyond this context.

In conclusion, to improve care transitions for $\mathrm{HHC}$ patients and their caregivers, emphasizing engagement of caregivers is key to ensure that they are educated about $\mathrm{HHC}$, provided with additional support as needed, and included in initial HHC 
visits once the patients are at home. Even though patients and caregivers with prior $\mathrm{HHC}$ experience often had clear expectations for $\mathrm{HHC}$, a strategy to uniformly engage caregivers across a range of experience can ensure caregivers have all the information and support needed to optimize care transitions to $\mathrm{HHC}$.

Disclosures: The authors have nothing to disclose.

Funding: Dr. Christine Jones is supported by grant number K08HS024569 from the Agency for Healthcare Research and Quality. Jason Falvey was supported by grant F31AG056069 from the National Institute on Aging, National Institutes of Health and is currently supported by T32AG019134. The content is solely the responsibility of the authors and does not necessarily represent the official views of the Agency for Healthcare Research and Quality or the National Institutes of Health.

\section{References}

1. Jones $C D$, Wald HL, Boxer RS, et al. Characteristics associated with home health care referrals at hospital discharge: results from the 2012 National Inpatient Sample. Health Serv Res. 2017;52(2):879-894. doi: 10.1111/1475-6773.

2. Avalere Health. Home Health Chartbook 2015: Prepared for the Alliance for Home Health Quality and Innovation. 2016.

3. Hospital Compare. https://www.medicare.gov/hospitalcompare/search. html. Accessed May 1, 2017.

4. Jones $C D, V u M B, O^{\prime}$ Donnell $C M$, et al. A failure to communicate: a qualitative exploration of care coordination between hospitalists and primary care providers around patient hospitalizations. J Gen Intern Med. 2015;30(4):417424. doi: 10.1007/s11606-014-3056-x.

5. Jones $C D$, Jones J, Richard A, et al. "Connecting the dots": a qualitative study of home health nurse perspectives on coordinating care for recently discharged patients. J Gen Intern Med. 2017;32(10):1114-1121. doi: 10.1007/ s11606-017-4104-0.

6. Foust JB, Vuckovic N, Henriquez E. Hospital to home health care transition: patient, caregiver, and clinician perspectives. West J Nurs Res. 2012;34(2):194212. doi: $10.1177 / 0193945911400448$.
7. Blair J, Volpe M, Aggarwal B. Challenges, needs, and experiences of recently hospitalized cardiac patients and their informal caregivers. J Cardiovasc Nurs. 2014;29(1):29-37. doi: 10.1097/JCN.0b013e3182784123.

8. Coleman EA, Roman SP. Family caregivers' experiences during transitions out of hospital. J Healthc Qual. 2015;37(1):12-21. doi: 10.1097/01. JHQ.0000460117.83437.b3.

9. Arbaje Al, Hughes A, Werner $\mathrm{N}$, et al. Information management goals and process failures during home visits for middle-aged and older adults receiving skilled home healthcare services after hospital discharge: a multisite, qualitative study. BMJ Qual Saf. 2018. doi: 10.1136/bmjqs-2018-008163.

10. Coleman EA. Family caregivers as partners in care transitions: the caregiver advise record and enable act. J Hosp Med. 2016;11(12):883-885. doi: 10.1002/jhm.2637.

11. Jones AL, Harris-Kojetin L, Valverde R. Characteristics and use of home health care by men and women aged 65 and over. Natl Health Stat Report. 2012(52):1-7.

12. Tian W. An all-payer view of hospital discharge to postacute care, 2013. HCUP Statistical Brief \#205. Rockville, Maryland; 2016.

13. McDonald KM, Sundaram V, Bravata DM, et al. Closing the Quality Gap: A Critical Analysis of Quality Improvement Strategies (Vol. 7: Care Coordination). Rockville, Maryland; 2007.

14. Coleman EA, Smith JD, Frank JC, Eilertsen TB, Thiare JN, Kramer AM. Development and testing of a measure designed to assess the quality of care transitions. Int J Integr Care. 2002;2:e02. doi: 10.5334/ijic.60.

15. Jones J, Nowels CT, Sudore R, Ahluwalia S, Bekelman DB. The future as a series of transitions: qualitative study of heart failure patients and their informal caregivers. J Gen Intern Med. 2015;30(2):176-182. doi: 10.1007/s11606-0143085-5.

16. Lum HD, Jones J, Lahoff $D$, et al. Unique challenges of hospice for patients with heart failure: a qualitative study of hospice clinicians. Am Heart J. 2015;170(3):524-530 e523. doi: 10.1016/j.ahj.2015.06.019

17. Kerr C, Nixon A, Wild D. Assessing and demonstrating data saturation in qualitative inquiry supporting patient-reported outcomes research. Expert Rev Pharmacoecon Outcomes Res. 2010;10(3):269-281. doi: 10.1586/ erp. 10.30

18. Sefcik JS, Nock RH, Flores EJ, et al. Patient preferences for information on post-acute care services. Res Gerontol Nurs. 2016;9(4):175-182. doi: 10.3928/19404921-20160120-01.

19. Coleman EA, Roman SP, Hall KA, Min SJ. Enhancing the care transitions intervention protocol to better address the needs of family caregivers. $J$ Healthc Qual. 2015;37(1):2-11. doi: 10.1097/01.JHQ.0000460118.60567.fe. 